\title{
EFFECTIVE MEASURES OF IRRATIONALITY FOR CERTAIN ALGEBRAIC NUMBERS
}

\author{
MICHAEL A. BENNETT
}

(Received 12 April 1996; revised 23 August 1996)

Communicated by W. W. Chen

\begin{abstract}
In this paper, we derive a number of explicit lower bounds for rational approximation to certain cubic irrationalities, proving, for example, that

$$
\left|\sqrt[3]{2}-\frac{p}{q}\right|>\frac{1}{4} q^{-2.5}
$$

for any non-zero integers $p$ and $q$. A number of these irrationality measures improve known results, including those for $\sqrt[3]{5}, \sqrt[3]{7}$ and $\sqrt[3]{11}$. Some Diophantine consequences are briefly discussed.

1991 Mathematics subject classification (Amer. Math. Soc.): primary 11J68, 11J82; secondary 11D25, 11 Y 50,11 Y 65.

Keywords and phrases: Effective irrationality measures, cubic irrationalities, Padé approximants, simultaneous figurate numbers.
\end{abstract}

\section{Introduction}

If $\theta$ is an algebraic number of degree $n$, then Liouville's theorem states that

$$
\left|\theta-\frac{p}{q}\right|>c(\theta) q^{-n}
$$

for any non-zero integers $p$ and $q$, where $c(\theta)$ is an effective constant. Improvements upon this have implications for the study of Diophantine equations. A stronger inequality was proven by Roth [18], who deduced that, if $\epsilon>0$, then

$$
\left|\theta-\frac{p}{q}\right|>c(\theta, \epsilon) q^{-2-\epsilon}
$$

Research supported by an NSERC Postdoctoral Fellowship

(C) 1997 Australian Mathematical Society $0263-6115 / 97$ \$A2.00+0.00 
for non-zero $p, q \in \mathbb{Z}$, where the constant $c(\theta, \epsilon)$ is, unfortunately, not computable. An effective improvement upon Liouville was, however, obtained by Baker [1, 2] for specific classes of algebraic numbers. In particular, he showed that

$$
\left|\sqrt[3]{2}-\frac{p}{q}\right|>10^{-6} q^{-2.955}
$$

for all positive integers $p$ and $q$, whence solutions of the equation

$$
x^{3}-2 y^{3}=u
$$

satisfy

$$
\max \{|x|,|y|\} \leq\left(3 \cdot 10^{5} \cdot|u|\right)^{23}
$$

By a more detailed analysis of certain Padé approximants involved in Baker's results, Chudnovsky [7] derived some effective improvements upon them. For example, he showed that

$$
\left|\sqrt[3]{2}-\frac{p}{q}\right|>c q^{-2.42971}
$$

for $p, q \in \mathbb{Z}$ with $q \neq 0$ and $c$ some effectively computable constant. The actual value of $c$, however, was not given. In 1986, Easton [8] produced explicit versions of a number of Chudnovsky's bounds for cubic irrationalities. Analogous to (1) and (2), he gave the inequality

$$
\left|\sqrt[3]{2}-\frac{p}{q}\right|>2.2 \times 10^{-8} q^{-2.795}
$$

for integers $p$ and $q(q \neq 0)$.

In [4], while studying simultaneous approximation to algebraic numbers, the author derived a result on approximation to a single algebraic number that is asymptotically equivalent to the aforementioned work of Chudnovsky. This follows work of Rickert [16] and, in the one dimensional case, is essentially Theorem 2 of Heimonen, et al. [10]. For the purposes of obtaining explicit bounds, this new approach has some computational advantages. Critical to Easton's estimates are upper and lower bounds due to McCurley [13] upon the function

$$
\theta(x, 3,2)=\sum_{\substack{p \leq x \\ p \equiv 2 \bmod 3}} \log p
$$


where the sum is over prime $p$. We obtain stronger results primarily because we are able to avoid consideration of $\theta(x, 3,2)$ and instead utilize sharper bounds due to Schoenfeld [19] (see also Rosser and Schoenfeld [17]) for the function

$$
\theta(x)=\sum_{p \leq x} \log p .
$$

Applying these techniques yields, analogous to (1) and (3), the inequality

$$
\left|\sqrt[3]{2}-\frac{p}{q}\right|>\frac{1}{4} q^{-2.5}
$$

for positive integers $p$ and $q$, which implies that

$$
\left|x^{3}-2 y^{3}\right| \geq \sqrt{x}
$$

for all non-negative integers $x$ and $y$. In the following, we describe the computations necessary to derive a slightly stronger version of (4) and related results for other cubic irrationalities. In particular, we prove

THEOREM 1.1. Suppose that $a$ and $N$ are positive integers satisfying

$$
8(\sqrt{N}+\sqrt{N+a})^{2}>a^{4} \kappa(a)^{3},
$$

where

$$
\kappa(a)= \begin{cases}3 \sqrt{3} & \text { if } \operatorname{ord}_{3} a=0 \\ \sqrt{3} & \text { if } \operatorname{ord}_{3} a=1 \\ 1 & \text { if } \operatorname{ord}_{3} a>1\end{cases}
$$

and that $p$ and $q$ are any positive integers. Then we have

$$
\left|\sqrt[3]{1+\frac{a}{N}}-\frac{p}{q}\right|>(4 \kappa(a) N)^{-1}\left(10^{4} q\right)^{-\lambda}
$$

where

$$
\lambda=1+\frac{\log \left(\kappa(a)(\sqrt{N}+\sqrt{N+a})^{2} / 2\right)}{\log \left(2(\sqrt{N}+\sqrt{N+a})^{2} / a^{2} \kappa(a)\right)} .
$$

We apply this result to produce explicit measures of irrationality for certain algebraic numbers of the form $\sqrt[3]{m}$ with $m \in \mathbb{Z}$, including those considered by Chudnovsky. Specifically, we show 
COROLLARY 1.2. If $p$ and $q$ are positive integers, then we have

$$
\left|\theta-\frac{p}{q}\right|>c(\theta) q^{-\lambda(\theta)}
$$

where we may take $\theta, c(\theta)$ and $\lambda(\theta)$ as follows:

\begin{tabular}{||ccc|ccc|ccc||}
\hline$\theta$ & $c(\theta)$ & $\lambda(\theta)$ & $\theta$ & $c(\theta)$ & $\lambda(\theta)$ & $\theta$ & $c(\theta)$ & $\lambda(\theta)$ \\
\hline$\sqrt[3]{2}$ & 0.25 & 2.47 & $\sqrt[3]{26}$ & 0.03 & 2.53 & $\sqrt[3]{63}$ & 0.02 & 2.43 \\
$\sqrt[3]{3}$ & 0.39 & 2.76 & $\sqrt[3]{28}$ & 0.03 & 2.52 & $\sqrt[3]{65}$ & 0.02 & 2.43 \\
$\sqrt[3]{5}$ & 0.29 & 2.80 & $\sqrt[3]{30}$ & 0.10 & 2.72 & $\sqrt[3]{66}$ & 0.04 & 2.50 \\
$\sqrt[3]{6}$ & 0.01 & 2.35 & $\sqrt[3]{31}$ & 0.14 & 2.97 & $\sqrt[3]{67}$ & 0.06 & 2.56 \\
$\sqrt[3]{7}$ & 0.08 & 2.70 & $\sqrt[3]{37}$ & 0.01 & 2.27 & $\sqrt[3]{68}$ & 0.08 & 2.60 \\
$\sqrt[3]{10}$ & 0.15 & 2.45 & $\sqrt[3]{39}$ & 0.09 & 2.21 & $\sqrt[3]{70}$ & 0.12 & 2.68 \\
$\sqrt[3]{11}$ & 0.22 & 2.91 & $\sqrt[3]{42}$ & 0.13 & 2.46 & $\sqrt[3]{76}$ & 0.10 & 2.54 \\
$\sqrt[3]{12}$ & 0.28 & 2.95 & $\sqrt[3]{43}$ & 0.01 & 2.32 & $\sqrt[3]{78}$ & 0.03 & 2.60 \\
$\sqrt[3]{13}$ & 0.35 & 2.86 & $\sqrt[3]{44}$ & 0.22 & 2.87 & $\sqrt[3]{83}$ & 0.10 & 2.72 \\
$\sqrt[3]{15}$ & 0.19 & 2.54 & $\sqrt[3]{52}$ & 0.26 & 2.97 & $\sqrt[3]{84}$ & 0.37 & 2.92 \\
$\sqrt[3]{17}$ & 0.01 & 2.22 & $\sqrt[3]{58}$ & 0.12 & 2.71 & $\sqrt[3]{90}$ & 0.09 & 2.41 \\
$\sqrt[3]{19}$ & 0.02 & 2.30 & $\sqrt[3]{60}$ & 0.08 & 2.61 & $\sqrt[3]{91}$ & 0.01 & 2.29 \\
$\sqrt[3]{20}$ & 0.01 & 2.23 & $\sqrt[3]{61}$ & 0.06 & 2.56 & & & \\
$\sqrt[3]{22}$ & 0.08 & 2.31 & $\sqrt[3]{62}$ & 0.04 & 2.50 & & & \\
\hline
\end{tabular}

Here, we have restricted ourselves to values of $\sqrt[3]{m}$ with $2 \leq m \leq 100$ which generate distinct cubic fields. These results sharpen those of Easton [8] in all cases and include new effective irrationality measures, in the range considered by Chudnovsky [7], for $\sqrt[3]{5}, \sqrt[3]{7}$ and $\sqrt[3]{11}$. While the techniques we utilize are applicable in a somewhat more general setting, we confine our attention to the special situation of cubic irrationalities for simplicity's sake. A different and much more general approach in this case is via linear forms in logarithms where Baker and Stewart [3] derived an explicit improvement upon Liouville's Theorem for all algebraic numbers of the form $\sqrt[3]{m}$ with $m$ not a cube. In the examples we deal with, however, the resulting irrationality measures are much stronger by our method. In Section 6, we will briefly discuss applications of these results to the solution of certain Diophantine equations, in particular, to those corresponding to simultaneous figurate numbers.

\section{Some technical preliminaries}

We begin, following Rickert [16], by constructing the diagonal Padé approximants to the function $(1+a x)^{1 / 3}$, for $a$ any positive integer. These are produced via 
consideration of the contour integral

$$
I_{l}(x)=\frac{1}{2 \pi i} \int_{\gamma} \frac{(1+z x)^{k+1 / 3}}{(z-l a)(z(z-a))^{k}} d z \quad(0 \leq l \leq 1)
$$

where $|x|<1 / a$ and $\gamma$ is a closed positively oriented contour enclosing both 0 and a. Cauchy's theorem then implies that

$$
I_{l}(x)=p_{l 0}(x)+(1+a x)^{1 / 3} p_{l 1}(x) \quad(0 \leq l \leq 1)
$$

where (see [16, Lemma 3.3]) we have

(6) $\quad p_{l m}(x)=a^{-2 k} \sum_{r=0}^{k_{l m}}(-1)^{m r+k_{l m}}\left(\begin{array}{c}k+1 / 3 \\ r\end{array}\right)\left(\begin{array}{c}2 k-r-1 \\ k_{l m}-r\end{array}\right)(a x)^{r}(1+a x m)^{k-r}$

with $k_{l m}=k-1+\delta_{l m}$ for $\delta_{l m}$ the Kronecker delta and $0 \leq l, m \leq 1$. By substituting $x=1 / N$ (for $N>a$ a positive integer), we obtain a sequence of 'good' rational approximations to $(1+a / N)^{1 / 3}$ and can utililize the following lemma to find an explicit measure of irrationality.

LEMMA 2.1. Suppose $\theta$ is real and that there exist positive real numbers $c, d, C$ and $D(D>1)$ such that for each positive integer $k$, we can find integers $p_{\text {lmk }}(0 \leq$ $l, m \leq 1)$ with non-zero determinant,

$$
\left|p_{l m k}\right| \leq c C^{k} \quad \text { and } \quad\left|p_{l 0 k}+p_{l 1 k} \theta\right| \leq d D^{-k} \quad(0 \leq l, m \leq 1) .
$$

Then we may conclude that

$$
\left|\theta-\frac{p}{q}\right|>\left(3 c C(\max \{1,1.5 d\})^{\log (C) / \log (D)}\right)^{-1} q^{-1-\log (C) / \log (D)}
$$

for all positive integers $p$ and $q$.

PROOF. This is a slight modification of a special case of [16, Lemma 2.1].

To apply this, for each positive integer $k$, we need to bound the quantities $\left|I_{l}(1 / N)\right|$ and $\left|p_{l m}(1 / N)\right|$ from above and to find a rational $\Psi_{k}$ such that $\Psi_{k} p_{l m}(1 / N)$ is an integer for $0 \leq l, m \leq 1$. Regarding the first of these problems, we prove

LEMMA 2.2. If $0 \leq l \leq 1$ and $N \geq 4 a$, then

$$
\left|I_{l}(1 / N)\right| \leq\left(N(\sqrt{N}+\sqrt{N+a})^{2}\right)^{-k}
$$


PROOF. Arguing as in [4, Lemma 3.2], we have

$$
\left|I_{l}(1 / N)\right|=\frac{\sqrt{3}}{2 \pi} N^{-2 k} \int_{0}^{\infty} \frac{x^{k+1 / 3} d x}{(x+1+a l / N)((x+1)(x+1+a / N))^{k}} .
$$

For $1 \leq k \leq 10$, we check, subject to $N \geq 4 a$, that

$$
\left|I_{l}(1 / N)\right| \leq\left(N(\sqrt{N}+\sqrt{N+a})^{2}\right)^{-k} .
$$

If $k>10$, then, noting that $\left|x^{1 / 3} /(x+1)\right| \leq \sqrt[3]{4} / 3$ for $x \geq 0$, (7) implies that

$$
\left|I_{l}(1 / N)\right| \leq \frac{\sqrt{3} \sqrt[3]{4}}{6 \pi} N^{-2 k} \int_{0}^{\infty}\left(\frac{x}{(x+1)(x+1+a / N)}\right)^{k} d x .
$$

We split this last integral into

$$
\int_{0}^{4}\left(\frac{x}{(x+1)(x+1+a / N)}\right)^{k} d x \text { and } \int_{4}^{\infty}\left(\frac{x}{(x+1)(x+1+a / N)}\right)^{k} d x
$$

and estimate them separately. The first of these is less than $4\left(N /(\sqrt{N}+\sqrt{N+a})^{2}\right)^{k}$. The second is no greater than

$$
\int_{4}^{\infty}\left(\frac{x}{(x+1)^{2}}\right)^{k} d x<\int_{5}^{\infty} x^{-k} d x
$$

and since $k>10$, this is less than $5^{-k}$. From $N \geq 4 a$, we have

$$
\frac{N}{(\sqrt{N}+\sqrt{N+a})^{2}} \geq \frac{4}{9+4 \sqrt{5}}>\frac{1}{5},
$$

whence

$$
\left|I_{l}(1 / N)\right| \leq \frac{5 \sqrt{3} \sqrt[3]{4}}{6 \pi}\left(N(\sqrt{N}+\sqrt{N+a})^{2}\right)^{-k}
$$

for $k>10$, and the result follows.

We also have

LEMMA 2.3. If $0 \leq l, m \leq 1$ and $N \geq 4 a$, then

$$
\left|p_{l m}(1 / N)\right| \leq 1.16\left((\sqrt{N}+\sqrt{N+a})^{2} / a^{2} N\right)^{k} .
$$


PROOF. The function $p_{l m}(x)(1+a m x)^{1 / 3}$ is given by the integral (5) with the contour changed so as to enclose the integer am but not $a(1-m)$. We may therefore write

$$
p_{l m}(1 / N)(1+a m / N)^{1 / 3}=\frac{1}{2 \pi i} \int_{\Gamma_{m}} \frac{(1+z / N)^{k+1 / 3}}{(z-l a)(z(z-a))^{k}} d z \quad(0 \leq l, m \leq 1)
$$

where $\Gamma_{0}$ and $\Gamma_{1}$ are defined by $|z|=\sqrt{N^{2}+a N}-N:=c_{0}$ and $|z-a|=N+a-$ $\sqrt{N^{2}+a N}:=c_{1}$ respectively. It follows that

$$
p_{l 0}(1 / N)=\frac{1}{2 \pi} \int_{-\pi}^{\pi}\left(\frac{c_{0} e^{i \theta}}{c_{0} e^{i \theta}-l a}\right) \frac{\left(1+c_{0} e^{i \theta} / N\right)^{k+1 / 3}}{\left(c_{0} e^{i \theta}\left(c_{0} e^{i \theta}-a\right)\right)^{k}} d \theta \quad(0 \leq l \leq 1)
$$

and the fact that $\left|c_{0} e^{i \theta}-a\right| \geq c_{1}>c_{0}$ implies

$$
\left|p_{l 0}(1 / N)\right| \leq\left(1+c_{0} / N\right)^{1 / 3}\left(\frac{1+c_{0} / N}{c_{0} c_{1}}\right)^{k} .
$$

Arguing similarly and using $c_{0} \leq\left|c_{1} e^{i \theta}+a\right| \leq c_{1}+a$ yields

$$
\left|p_{l 1}(1 / N)\right| \leq \frac{c_{1}}{c_{0}}\left(1+c_{1} /(N+a)\right)^{1 / 3}\left(\max _{-\pi \leq \theta \leq \pi}\left|\frac{1+\left(c_{1} e^{i \theta}+a\right) / N}{c_{1}\left(c_{1} e^{i \theta}+a\right)}\right|\right)^{k} .
$$

By calculus, this is no greater than

$$
\frac{c_{1}}{c_{0}}\left(1+c_{1} /(N+a)\right)^{1 / 3}\left(\frac{1+c_{0} / N}{c_{0} c_{1}}\right)^{k} .
$$

Since

$$
\left(1+c_{0} / N\right) / c_{0} c_{1}=(\sqrt{N}+\sqrt{N+a})^{2} / a^{2} N
$$

and assuming $N \geq 4 a$, we have

$$
\max \left\{\left(1+\frac{c_{0}}{N}\right)^{1 / 3}, \frac{c_{1}}{c_{0}}\left(1+\frac{c_{1}}{N+a}\right)^{1 / 3}\right\}=\frac{c_{1}}{c_{0}}\left(1+\frac{c_{1}}{N+a}\right)^{1 / 3}<1.16
$$

and the result obtains.

\section{Arithmetic properties of $p_{l m}(1 / N)$}

Define, for positive integers $j$ and $k$, the intervals $I_{j k}$ by $I_{j k}=[(k+1) / j,(3 k-$ 4) $/(3 j-1)]$. Also, for $0 \leq s \leq k$, let

$$
G(s)=\operatorname{gcd}\left\{3^{[3 s / 2]}\left(\begin{array}{c}
k+1 / 3 \\
r
\end{array}\right)\left(\begin{array}{c}
2 k-r-1 \\
s-r
\end{array}\right): r=0,1, \ldots s\right\}
$$


where this latter expression is well defined since [7, Lemma 4.2] gives that $3^{[3 s / 2]}\left(\begin{array}{c}k+1 / 3 \\ r\end{array}\right)$ $\in \mathbb{Z}$ for $0 \leq r \leq s$ and $k \in \mathbb{N}$. If we let $G_{k}=\operatorname{gcd}\{G(k), G(k-1)\}$ then we have

LEMMA 3.1. Suppose that $j$ and $k$ are positive integers with $1 \leq j \leq \sqrt{k / 3}$. Then if $p$ is prime with $p \in I_{j k}$, it follows that $p$ divides $G_{k}$.

ProOF. We prove that such primes divide $G(k)$, the case with $G(k-1)$ being similar. From $p \in I_{j k}$, we have

$$
(3 j-1)\left(\frac{k-1}{3 k-4}\right) \leq \frac{k-1}{p} \leq j\left(\frac{k-1}{k+1}\right)
$$

and thus $\{(k-1) / p\}>2 / 3$, where $\{x\}$ denotes the fractional part of $x$. Since $1 \leq j \leq \sqrt{k / 3}$, one may conclude that $p>\sqrt{3 k+1}$ and so, if $0 \leq r \leq k$,

$$
\operatorname{ord}_{p}\left(\begin{array}{c}
2 k-r-1 \\
k-r
\end{array}\right)=\left\{\frac{k-1}{p}\right\}+\left\{\frac{k-r}{p}\right\}-\left\{\frac{2 k-r-1}{p}\right\} .
$$

If $p$ does not divide $\left(\begin{array}{c}2 k-r-1 \\ k-r\end{array}\right)$, then $\{(k-r) / p\}<1-\{(k-1) / p\}$ whence, since $\{(k-1) / p\}>2 / 3$ and $p$ does not divide $k$,

$$
\left\{\frac{r}{p}\right\} \geq\left\{\frac{k}{p}\right\}+\left\{\frac{k-1}{p}\right\}+\frac{1}{p}-1 .
$$

By [7, Lemma 4.5], using $p>\sqrt{3 k+1}$, we have

$$
\operatorname{ord}_{p}\left(\begin{array}{c}
k+1 / 3 \\
r
\end{array}\right)=\left\{\frac{k-r-\theta}{p}\right\}+\left\{\frac{r}{p}\right\}-\left\{\frac{k-\theta}{p}\right\}
$$

where $\theta=(p q-1) / 3$ for $1 \leq q \leq 2$ satisfying $p q \equiv 1 \bmod 3$. Inequality (8) then implies that

$$
\left\{\frac{r}{p}\right\}-\left\{\frac{k-\theta}{p}\right\} \geq\left\{\frac{k-1}{p}\right\}+\frac{1}{p}-\frac{2}{3}-\frac{1}{3 p} .
$$

Since this is greater than 0 , we have $\operatorname{ord}_{p}(\underset{r}{k+1 / 3}) \geq 1$ as desired.

We use this result to show

LEMMA 3.2. $G_{k}>(1 / 5563) 2^{k}$ for $k \geq 1$.

PROOF. Suppose, first, that $k \geq 220000$. From Schoenfeld [19], we have that

$$
\theta(x)=\sum_{p \leq x} \log p<1.000081 x \quad(x>0)
$$


where the sum is over prime $p$. For analogous lower bounds upon $\theta(x)$, we utilize $[19$, Corollary $2 *]$. Define $L_{l, k}=\sum_{p \in I_{l k}} \log p$. It follows that

$$
\begin{aligned}
& L_{1, k}>0.49584 k-1.99458, \\
& L_{2, k}>0.09728 k-0.79643
\end{aligned}
$$

and

$$
L_{3, k}>0.03943 k-0.49706 .
$$

We derive similar inequalities for $L_{l, k}$ for $4 \leq l \leq 18$ and, summing them, may thus apply Lemma 3.1 to conclude that

$$
\log G_{k}>0.69493 k-5.58728>k \log 2
$$

so that $G_{k}>2^{k}$ for $k \geq 220000$.

For $1 \leq k \leq 500$, we compute $G_{k}$ directly from the definition and note that in all cases

$$
G_{k} / 2^{k}>1 / 5563
$$

where $G_{k} / 2^{k}$ is minimal for $k=105$. For each $k$ with $500<k \leq 5000$, we compute

$$
P_{k}=\prod_{1 \leq j \leq \sqrt{k / 3}} \prod_{p \in I_{j k}} p
$$

and find that

$$
P_{k} / 2^{k}>1 / 5563
$$

with only 222 exceptions, the last being with $k=1581$. For each of these, we calculate $G_{k}$ and check that, again, inequality (9) is satisfied.

To tackle the cases with $5000<k<220000$, we define

$$
Q_{k, r}=\prod_{1 \leq j \leq \sqrt{k / 3}} \prod_{\frac{k+1}{j} \leq p \leq \frac{k+r}{j}} p \quad(r \geq 1)
$$

and note that $P_{k+l} \geq P_{k} / Q_{k, r}$ for $1 \leq l \leq r$. This observation enables us to reduce the roughly 215000 possibilities for $k$ to a few hundred. For example, if we calculate $P_{100000}$, we find that $\log \left(P_{100000} / 2^{100000}\right)>4582$ while $\log \left(Q_{100000,798}\right)<4587$ so that (10) (and hence (9)) is satisfied for $100000 \leq k<100798$. Somewhat crudely, this permits us to check every 20th value for $k$ with $5000<k \leq 9000$, every 50th $k$ for $9000<k \leq 15000$, every 100 th $k$ for $15000<k \leq 26000$, every 200 th $k$ 
for $26000<k \leq 38000$, every 300 th $k$ for $38000<k \leq 63000$, every 500th $k$ for $63000<k \leq 134000$ and every 1000th $k$ for $134000<k \leq 220000$. Performing these calculations, using Maple $\mathrm{V}$, and verifying inequality (10) in all cases completes the proof.

We note that Lemma 3.2 may be improved somewhat, since one may in fact show that

$$
\lim _{k \rightarrow \infty} G_{k}^{1 / k}=3 \sqrt{3} e^{-\pi \sqrt{3} / 6}=2.09807 \ldots
$$

\section{Proof of Theorem 1.1 and Corollary 1.2}

We first note that the condition

$$
8(\sqrt{N}+\sqrt{N+a})^{2}>a^{4} \kappa(a)^{3}
$$

implies, since $a$ and $N$ are positive integers, that $N \geq 4 a$. From (6) and the observation that $3^{[3 r / 2]}\left(\begin{array}{c}k+1 / 3 \\ r\end{array}\right)$ is an integer for $0 \leq r \leq k$, if we let

$$
\Psi_{k}=N^{k} a^{2 k} 3^{\max \left[[3 k / 2]-k \operatorname{ord}_{3} a, 0\right]} G_{k}^{-1},
$$

then, setting $p_{l m k}=\Psi_{k} p_{l m}(1 / N)$, we have that $p_{l m k} \in \mathbb{Z}$ for $0 \leq l, m \leq 1$. Furthermore, [16, Lemma 3.4] gives that $\operatorname{det}\left(p_{l m k}\right)$ is non-zero for each positive integer $k$. Lemmas 2.3 and 3.2 imply, then, that

$$
\left|p_{l m k}\right| \leq 6454\left(\frac{\kappa(a)}{2}(\sqrt{N}+\sqrt{N+a})^{2}\right)^{k} .
$$

Since Lemmas 2.2 and 3.2 yield

$$
\left|p_{l 0 k}+p_{l 1 k} \sqrt[3]{1+\frac{a}{N}}\right| \leq 5563\left(\frac{2}{a^{2} \kappa(a)}(\sqrt{N}+\sqrt{N+a})^{2}\right)^{-k}
$$

we may apply Lemma 2.1 to conclude that

$$
\left|\sqrt[3]{1+\frac{a}{N}}-\frac{p}{q}\right|>c q^{-\lambda}
$$

for positive integers $p$ and $q$, where $\lambda$ is as in the statement of the theorem and

$$
c^{-1}=9681(8344.5)^{\lambda-1} \kappa(a)(\sqrt{N}+\sqrt{N+a})^{2} .
$$

Utilizing $N \geq 4 a$ yields $c^{-1}<4 \kappa(a) N 10^{4 \lambda}$ which completes the proof. 
We illustrate the proof of Corollary 1.2 with an effective irrationality measure for $\sqrt[3]{2}$. Taking $N=125$ and $a=3$ (so that $\kappa(a)=\sqrt{3}$ ) in Theorem 1.1 implies that

$$
\left|\frac{4}{5} \sqrt[3]{2}-\frac{4 p}{5 q}\right|>(500 \sqrt{3})^{-1}(50000 q)^{-\lambda}
$$

where

$$
\lambda=1+\frac{\log \left(\frac{\sqrt{3}}{2}(253+80 \sqrt{10})\right)}{\log \left(\frac{2}{9 \sqrt{3}}(253+80 \sqrt{10})\right)}=2.45758 \ldots
$$

It follows that

$$
\left|\sqrt[3]{2}-\frac{p}{q}\right|>\left(2.448 \times 10^{14}\right)^{-1} q^{-\lambda}
$$

for $q \geq 1$, so we have

$$
\left|\sqrt[3]{2}-\frac{p}{q}\right|>\frac{1}{4} q^{-2.47}
$$

provided $q \geq 10^{1111}$.

To check the cases $1 \leq q<10^{1111}$, we note that if $p$ and $q$ fail to satisfy (12), then $p / q$ must be a convergent in the continued fraction expansion to $\sqrt[3]{2}$ (see [11, Theorem 9.7]). Computing the first 3000 such convergents using Maple V, we have that only the first 2208 have denominators $<10^{1111}$. If we denote the $i$ th convergent to $\sqrt[3]{2}$ by $p_{i} / q_{i}$ and the $i$ th partial quotient by $a_{i}$, then (see [11, Theorem 9.6])

$$
\left|\sqrt[3]{2}-\frac{p_{i}}{q_{i}}\right|>\frac{1}{\left(a_{i+1}+2\right) q_{i}^{2}}
$$

whence, if $p_{i} / q_{i}$ does not satisfy (12), we require

$$
a_{i+1}>4 q_{i}^{0.47}-2 \text {. }
$$

Checking that the first 100 convergents satisfy (12) and noting that $q_{101}>10^{56}$, from (13) we need only show that $a_{j}<10^{26}$ for $102 \leq j \leq 2209$ to reach the desired conclusion. Since the largest value for $a_{j}$ in the range in question is attained by $a_{1991}=12737$, we conclude as stated.

We argue in a similar fashion to handle the other examples in Corollary 1.2. In each case, to obtain the bounds cited in Corollary 1.2, we are led to consider no more than 3100 convergents in the related continued fraction expansions and find no partial quotients exceeding 49968 (the 813th partial quotient to $\sqrt[3]{5}$ ). In the following table, we list the choices of $a$ and $N$ for Theorem 1.1 which generate the examples in Corollary 1.2 : 


\begin{tabular}{|c|c|c|c|c|c|}
\hline$\theta$ & $a, N$ & $\theta$ & $a, N$ & $\theta$ & $a, N$ \\
\hline$\sqrt[3]{2}$ & $3,5^{3}$ & $\sqrt[3]{26}$ & 1,26 & $\sqrt[3]{62}$ & 1,31 \\
$\sqrt[3]{3}$ & $1,2^{3}$ & $\sqrt[3]{28}$ & $1,3^{3}$ & $\sqrt[3]{63}$ & 1,63 \\
$\sqrt[3]{5}$ & $402657,5(140145707)^{3}$ & $\sqrt[3]{30}$ & $1,3^{2}$ & $\sqrt[3]{65}$ & $1,4^{3}$ \\
$\sqrt[3]{6}$ & $5,6(257)^{3}$ & $\sqrt[3]{31}$ & $15,31(7)^{3}$ & $\sqrt[3]{66}$ & $1,2(4)^{2}$ \\
$\sqrt[3]{74}$ & $15,7(23)^{3}$ & $\sqrt[3]{37}$ & $1,37(3)^{3}$ & $\sqrt[3]{67}$ & $3,4^{3}$ \\
$\sqrt[3]{10}$ & $9,14(28)^{2}$ & $\sqrt[3]{39}$ & $1,23^{3}$ & $\sqrt[3]{68}$ & $1,4^{2}$ \\
$\sqrt[3]{11}$ & $1887,11(11251)^{3}$ & $\sqrt[3]{42}$ & $1,6(2)^{3}$ & $\sqrt[3]{70}$ & $3,2(4)^{2}$ \\
$\sqrt[3]{12}$ & $13,4(38)^{3}$ & $\sqrt[3]{43}$ & $1,7^{3}$ & $\sqrt[3]{76}$ & $35,2(2353)^{3}$ \\
$\sqrt[3]{13}$ & $14,13(37)^{3}$ & $\sqrt[3]{44}$ & $9,7^{3}$ & $\sqrt[3]{78}$ & $5,78(11)^{3}$ \\
$\sqrt[3]{15}$ & $1,3(2)^{3}$ & $\sqrt[3]{52}$ & 3,13 & $\sqrt[3]{83}$ & $19,253^{3}$ \\
$\sqrt[3]{17}$ & $1,17(7)^{3}$ & $\sqrt[3]{58}$ & 3,29 & $\sqrt[3]{84}$ & $2927,148273^{3}$ \\
$\sqrt[3]{19}$ & $1,8^{3}$ & $\sqrt[3]{60}$ & 1,15 & $\sqrt[3]{90}$ & $1,10(2)^{3}$ \\
$\sqrt[3]{20}$ & $1,19^{3}$ & $\sqrt[3]{61}$ & 3,61 & $\sqrt[3]{91}$ & $1,91(2)^{3}$ \\
$\sqrt[3]{22}$ & $3,7(14)^{2}$ & & & & \\
\hline
\end{tabular}

\section{Applicability of Theorem 1.1}

If one is content with effective irrationality measures, rather than explicit, then the best result in our situation, due to Chudnovsky [7] (see also [4, 10]), is essentially Theorem 1.1 with the constant 2 appearing in the expression for $\lambda$ replaced by $3 \sqrt{3} e^{-\pi \sqrt{3} / 6}$ (which is roughly $2.098 \ldots$ ). This implies new irrationality measures of the form

$$
\begin{array}{ll}
\left|\sqrt[3]{5}-\frac{p}{q}\right|>c_{1} q^{-2.75567}, & \left|\sqrt[3]{7}-\frac{p}{q}\right|>c_{2} q^{-2.66974} \\
\left|\sqrt[3]{11}-\frac{p}{q}\right|>c_{3} q^{-2.87124}, & \left|\sqrt[3]{41}-\frac{p}{q}\right|>c_{4} q^{-2.99342}
\end{array}
$$

where $c_{1}, c_{2}, c_{3}$ and $c_{4}$ are effectively computable constants. The first three of these correspond to the choices of $a$ and $N$ given in the previous table while the last follows from taking $a=51$ and $N=41(29)^{3}$. Theorem 1.1 also yields the result

$$
\left|\sqrt[3]{57}-\frac{p}{q}\right|>\left(2.2 \times 10^{25}\right)^{-1} q^{-2.99738}
$$

for integers $p$ and $q(q \neq 0)$, by taking $a=26$ and $N=127^{3}$. We omit this from Corollary 1.2 since it requires a rather more detailed analysis of the related continued fraction expansion to reduce the coefficient $2.2 \times 10^{25}$ to something more pleasant. 
To generate these examples, we consider convergents from the continued fraction expansion to $\sqrt[3]{m}$. If we are able to find a convergent $p / q$ satisfying, roughly,

$$
\left|\sqrt[3]{m}-\frac{p}{q}\right|<m^{-5 / 12} q^{-9 / 4}
$$

then we may apply Theorem 1.1 with

$$
1+\frac{a}{N}=\frac{p^{3}}{m q^{3}} \quad \text { or } \quad \frac{m q^{3}}{p^{3}}
$$

to deduce an irrationality measure for $\sqrt[3]{m}$. In each of the cases dealt with in Corollary 1.2 , such an approximation occurs among the first 20 convergents. For other examples, however, we need to search somewhat further; for $\sqrt[3]{200}$ the 27 th convergent is required, for $\sqrt[3]{826}$, the 25 th, etcetera. All in all, we are able to apply these techniques to derive effective irrationality measures for 42 of the 74 values for $\sqrt[3]{m}$ which generate distinct cubic fields with $2 \leq m \leq 100$ and for 233 of the 788 such $\sqrt[3]{m}$ with $2 \leq m \leq 1000$. The smallest $\sqrt[3]{m}$ for which we cannot apparently utilize Theorem 1.1 is with $m=14$, where none of the first 2000 convergents yield the desired approximation.

If we define $N(x)$ to be the number of positive integers $m \leq x$ for which Theorem 1.1 yields an effective improvement upon Liouville's Theorem for $\sqrt[3]{m}$, then we may readily show that $N(x) \gg x^{7 / 12}$ for $x \geq 2$. To see this, we note that if $M$ is any positive integer and $a \in \mathbb{N}$ satisfies $a \leq 2 M^{3 / 4} / 3$, then we may apply Theorem 1.1 to produce non-trivial measures of irrationality for both $\sqrt[3]{\left(M^{3}+a\right) / M^{3}}$ and $\sqrt[3]{M^{3} /\left(M^{3}-a\right)}$. This implies, in turn, non-trivial measures for all $\sqrt[3]{m}$ with $\left|M^{3}-m\right| \leq 2 M^{3 / 4} / 3$, so that we have

$$
N(x) \geq \sum_{M=2}^{\left[x^{1 / 3}\right]-1} 2\left[2 M^{3 / 4} / 3\right]
$$

whence, since

$$
\sum_{M \leq x} M^{3 / 4}=\frac{4 x^{7 / 4}}{7}+O\left(x^{3 / 4}\right)
$$

the result obtains.

\section{Applications to diophantine equations}

By application of Theorem 1.1 and Corollary 1.2, we are able to show 
THEOREM 6.1. If $x$ and $y$ are integers, then

$$
\begin{aligned}
& \left|x^{3}-2 y^{3}\right| \geq \max \{|x|,|y|\}^{0.53}, \\
& \left|x^{3}-3 y^{3}\right| \geq \max \{|x|,|y|\}^{0.24}, \quad \text { and } \\
& \left|x^{3}-6 y^{3}\right| \geq \max \{|x|,|y|\}^{0.65}
\end{aligned}
$$

where the last inequality holds unless $|x|=467$ and $|y|=257$.

PROOF. We note that the above inequalities are trivial if $\min \{|x|,|y|\} \leq 1$. Otherwise, we consider the cases

$$
|x-\sqrt[3]{m} y|<1 / \sqrt[3]{m} y \quad \text { and } \quad|x-\sqrt[3]{m} y| \geq 1 / \sqrt[3]{m} y
$$

separately and show that, for $m=2$ and $m=3$, the inequalities follow directly from Corollary 1.2 and the factorization

$$
x^{3}-m y^{3}=(x-\sqrt[3]{m})\left(x^{2}+\sqrt[3]{m} x y+\sqrt[3]{m^{2}} y^{2}\right) .
$$

In the case $m=6$, we can utilize Theorem 1.1 and a slightly more detailed analysis of the continued fraction expansion to $\sqrt[3]{6}$ to show that

$$
\left|\sqrt[3]{6}-\frac{p}{q}\right|>10 q^{-2.35}
$$

provided $q>257$. Checking the values $2 \leq q \leq 256$ yields the stated conclusion.

We apply these inequalities to solve a trio of problems on simultaneous figurate numbers. Let us define, for $m$ and $n$ integers, the sequences

$$
Q_{m}=\frac{m(m+1)(2 m+1)}{6}
$$

and

$$
T_{n}=\frac{n(n+1)(n+2)}{6} .
$$

The first of these is just the sum of the first $m$ squares and is known as the $m$ th square pyramidal number, while the second is the sum of the first $n$ triangular numbers and is called the $n$th tetrahedral number. They measure the number of spheres stacked in a pyramid with a square or triangular base, respectively. We show

THEOREM 6.2. If $m, n$ and $r$ are positive integers, then 
(i) if $Q_{m}=T_{n}$, then $m=n=1$

(ii) if $Q_{m}=r^{3}$, then $m=r=1$; and

(iii) if $T_{n}=r^{3}$, then $n=r=1$.

None of these results are new; in fact, parts (ii) and (iii) of the above theorem date back to Lucas [12] and Moret-Blanc [14] (1881) while part (i) was stated as a problem in the American Mathematical Monthly in 1940 and solved by Finkelstein (Ray Steiner) [9] in 1966, using a variety of number theoretical techniques. Independently, Beukers and Top [5] in 1988 proved (i) using an inequality similar to those in Theorem 6.1. Following [5] and [9], we may change variables so that solutions in integers to equations (i), (ii) or (iii) correspond to integral solutions to the systems of equations

(a) $x^{3}-2 y^{3} \in\{-2,-6,-10,-30\}$,

(b) $x^{3}-3 y^{3} \in\{1,3\}$, or

(c) $x^{3}-6 y^{3} \in\{1,2,3,6\}$

respectively. For example, if $Q_{m}=r^{3}$, then

$$
2 m(2 m+1)(2 m+2)=3(2 r)^{3}
$$

whence, taking $u=2 m+1$ and $v=2 r$, we have $u^{3}-u=3 v^{3}$. Therefore, either $u=w^{3}$ or $u=3 w^{3}$, the first of which leading to the equation $x^{3}-3 y^{3}=1$ with $x=w^{2}$ and $y=v / w$, while the second yields $x^{3}-3 y^{3}=3$ for $x=3 w^{2}$ and $y=v / w$. Applying Theorem 6.1 to the system of equations (a) implies that all solutions satisfy $\max \{|x|,|y|\} \leq 612$. Similarly, all solutions to (b) satisfy $\max \{|x|,|y|\} \leq 97$ while all those to (c) have $\max \{|x|,|y|\} \leq 15$. Checking beneath these bounds, we find that the only integer solutions $(x, y)$ to $(a)$ are $(0,1),(-2,-1),(-4,-3)$ and $(-2,1)$, while those to (b) and (c) are given by $(0,-1),(1,0)$ and $(3,2)$, and by $(0,-1),(1,0)$ and $(2,1)$, repectively. Working back through our various changes of variables, these imply that the integral solutions to $Q_{m}=T_{n}$ are given by $(m, n)=(-2,-3)$, $(-1,-2),(-1,-1),(-1,0),(0,-2),(0,-1),(0,0)$ and $(1,1)$. Similarly, the integral solutions to $Q_{m}=r^{3}$ are given by $(m, r)=(-2,-1),(-1,0),(0,0)$ and $(1,1)$ while those to $T_{n}=r^{3}$ are $(n, r)=(-3,-1),(-2,0),(-1,0),(0,0)$ and $(1,1)$. Theorem 6.2 therefore follows immediately.

\section{Concluding remarks}

As previously mentioned, these results may be extended to rather wider classes of algebraic numbers than those discussed here. We can, for instance, provide explicit bounds for rational approximation to fourth or sixth roots of rationals in a completely analogous fashion (that is, using only bounds from the work of Rosser and Schoenfeld upon the common factors of the coefficients of the approximating polynomials). For 
algebraic numbers of higher degree or of degree five, however, we need to utilize bounds upon primes in arithmetic progressions, say via recent work of Ramaré and Rumely [15].

\section{Acknowledgements}

The author would like to thank Professor Cameron Stewart for helpful suggestions on the format of this paper.

\section{References}

[1] A. Baker, 'Rational approximations to certain algebraic numbers', Proc. London Math. Soc. 14 (1964), 385-398.

[2] _ , 'Rational approximations to $\sqrt[2]{2}$ and other algebraic numbers', Quart. J. Math. Oxford Ser. (2) 15 (1964), 375-383.

[3] A. Baker and C. L. Stewart, 'On effective approximation to cubic irrationals', in: New advances in transcendence theory (Cambridge University Press, Cambridge, 1988) pp. 1-24.

[4] M. Bennett, 'Simultaneous rational approximation to binomial functions', Trans. Amer. Math. Soc. 348 (1996), 1717-1738.

[5] F. Beukers and J. Top, 'On oranges and integral points on certain plane cubic curves', Nieuw Arch. Wisk. (4) 6 (1988), 203-210.

[6] E. Bombieri and J. Mueller, 'On effective measures of irrationality for $(a / b)^{1 / r}$ and related numbers', J. Reine Angew. Math. 342 (1983), 173-196.

[7] G. V. Chudnovsky, 'On the method of Thue-Siegel', Ann. of Math. 117 (1983), 325-382.

[8] D. Easton, 'Effective irrationality measures for certain algebraic numbers', Math. Comp. 46 (1986), 613-622.

[9] R. Finkelstein, 'On a Diophantine equation with no non-trivial integral solution', Amer. Math. Monthly 73 (1966), 471-477.

[10] A. Heimonen, T. Matala-aho and K. Väänänen, 'An application of Jacobi type polynomials to irrationality measures’, Bull. Austral. Math. Soc. 50 (1994), 225-243.

[11] W. J. LeVeque, Topics in number theory (Addison-Wesley, Reading, 1956).

[12] E. Lucas, 'Problem', in: Nouv. Ann. Math. 15 (1876), 144.

[13] K. S. McCurley, 'Explicit estimates for $\theta(x ; 3 ; l)$ and $\psi(x ; 3 ; l)$ ', Math. Comp. 42 (1984), 287-296.

[14] M. Moret-Blanc, 'Solution to problem', in: Nouv. Ann. Math. 20 (1881) pp. 330-332.

[15] O. Ramaré and R. Rumely, 'Primes in arithmetic progressions', Math. Comp. 65 (1996), 397-425.

[16] J. H. Rickert, 'Simultaneous rational approximations and related diophantine equations', Proc. Cambridge Philos. Soc. 113 (1993), 461-472.

[17] J. Rosser and L. Schoenfeld, 'Sharper bounds for the Chebyshev functions $\theta(x)$ and $\psi(x)$ ', Math. Comp. 29 (1975), 243-269.

[18] K. F. Roth, 'Rational approximations to algebraic numbers', Mathematika 2 (1955), 1-20.

[19] L. Schoenfeld, 'Sharper bounds for the Chebyshev functions $\theta(x)$ and $\psi(x)$ II', Math. Comp. 30 (1976), 337-360.

\section{Department of Mathematics}

University of Michigan

Ann Arbor, MI 48109

USA

e-mail: mabennet@math.Isa.umich.edu 